\title{
The Risk/Benefit Analysis of Oral Anticoagulant Treatment in Atrial Fibrillation Using HAS-BLED and CHA2DS2-VASc Score
}

Silivastru (Cozlea) Ionela¹, Cozlea Daniel Laurentiu, Keresztesi Arthur Attila², Asofie Gabriela ${ }^{3}$, Cozlea Laurentiu ${ }^{1}$, Oltean Galafteon ${ }^{4}$, Dobru Daniela ${ }^{5}$

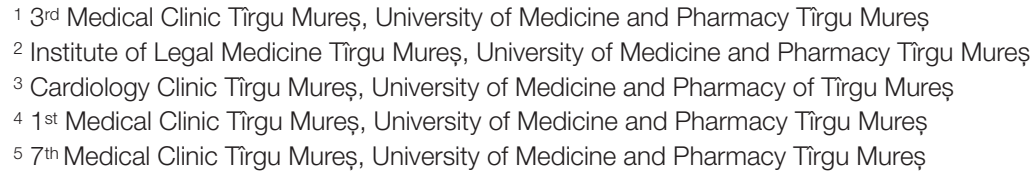

Introduction. The purpose of this study is to evaluate the risk and the benefit of oral anticoagulant treatment in nonvalvular atrial fibrillation (AF) patients, using the two scores recommended by the guidelines: the CHA2DS2-VASc score and HAS-BLED score.

Material and method. We conducted a retrospective observational study on 144 patients with nonvalvular atrial fibrillation, admitted between 1st of July 2013 and 30th September 2013 in the 3rd Medical Clinic of Tîrgu Mureș with a prospectiv follow-up at 6 months.

Based on the data collected from the patient charts, the thromboembolic risk was assessed using the CHA2DS2-VASc score and the hemorrhage risk was assessed using the HAS-BLED score. At 6 months, the patients were contacted via telephone and were questioned regarding their state of health, the existence of hospitalizations in the last 6 months, the international normalized ratio (INR) value, the existence of hemorrhagic or thromboembolic events.

Results. The group of patients was composed of 70 female and 74 male with the mean age of $70 \pm 11$ years. From the total number of patients $13(11.7 \%)$ had a history of stroke and the CHA2DS2-VASc score revealed that these 13 were in the high risk class. The presence of arterial hypertension and vascular disease were statistically associated with stroke.

Hemorrhagic events were encountered in 19 patients (13.19\%) and 16 of them had a higher than 3 HAS-BLED score. A history of bleeding, anemia and labile INR were the factors statistically associated with bleeding.

Conclusions. The CHA2DS2-VASc score is useful in stratifying patients with AF in risk groups for thromboembolic events while the HASBLED score proved to be a useful tool in predicting bleeding events in anticoagulated patients.

Keywords: atrial fibrillation, thromboembolic events, bleeding events, oral anticoagulant treatment.

Received: 09 December 2014 / Accepted: 04 June 2015

\section{Introduction}

Atrial fibrillation (AF) patients have a 5 fold higher thromboembolic risk than the general population, being estimated that $15 \%$ to $20 \%$ of ischemic strokes can be attributed to this arrhythmia [1].

Ischemic stroke due to atrial fibrillation has a higher mortality rate and the surviving patients remain with important neurological problems and have a higher rate of recurrence in comparison with patients who suffered an ischemic stroke without correlation with AF [2].

In a 2007 published meta-analysis, the relative risk of thromboembolic event rate in AF patients was reduced due to antithrombotic therapy. In ischemic stroke patients, this risk was reduced by $60 \%$ after antithrombotic therapy [3]. (Table I)

According to the newest AF guideline, for a $\mathrm{CHA}_{2} \mathrm{DS}_{2}$ VASc score of 0 no antithrombotic therapy should be given; in the case of patients with $\mathrm{CHA}_{2} \mathrm{DS}_{2}$-VASc score of 1 ,

* Correspondence to: Gabriela Asofie

E-mail: gabriela_asf@yahoo.com oral anticoagulant (OAC) therapy is recommended, after the bleeding risk is assessed; for a $\mathrm{CHA}_{2} \mathrm{DS}_{2}$-VASc score $\geq$ 2, OAC treatment is recommended, if possible [4].

The most important side effects of OAC therapy, especially in the vitamin K antagonist (VKAs) class (warfarin/ acenocumarol), are bleeding complications. From a clinical point of view, hemorrhagic episodes correlated with VKA treatment were divided into two major categories: minor and major bleeding events. Major hemorrhage consists in

Table I. Risk factors associated with thromboembolic events in AF patients (the $\mathrm{CHA}_{2} \mathrm{DS}_{2}$-VASc score)

\begin{tabular}{lc}
\hline Risk factors & Score \\
\hline Congestive heart failure & 1 \\
Hypertension & 1 \\
Age $\geq 75$ years & 2 \\
Diabetes mellitus & 1 \\
Stroke or TIA & 2 \\
Vascular disease & 1 \\
Age 65-74 years & 1 \\
Sex (female) & 1 \\
Maximum score & 9 \\
\hline
\end{tabular}


any hemorrhagic event in need of hospitalization or that led to a hemoglobin level drop with more than $2 \mathrm{mg} / \mathrm{L}$ or any episode that required blood transfusions [5].

The most serious OAC treatment complications is intracerebral bleeding, that has a poor prognosis, mainly due to the risk of extension [6,7].

Gastrointestinal (GI) bleeds are more frequent than intracerebral bleeding events, especially in patients that use OAC treatment associated with antiplatelet therapy. This association leads to a 13 fold increase of the hemorrhagic risk, especially in elderly [8]. Minor bleeding events are defined as any hemorrhage without major clinical impact on the patient's wellbeing, or which produces only an asymptomatic drop of hemoglobin levels [5].

The bleeding risk assessment is recommended before $\mathrm{OAC}$ treatment in AF patients, due the numerous associated comorbidities, that could lead to a higher bleeding risk (Table II).

\section{Table II. Bleeding risk factors (the HAS-BLED score)}

\begin{tabular}{lc}
\hline Risk factor & Score \\
\hline Hypertension & 1 \\
Renal or liver insufficiency & $1 / 2$ \\
History of stroke or TIA & 1 \\
History of bleeding & 1 \\
Labile INR & 1 \\
Age> 65 years & 1 \\
Drugs, alcohol & $1 / 2$ \\
Maximum score & 9 \\
\hline
\end{tabular}

The guidelines recommend that a patient with a 2 or higher HAS-BLED score should be periodically monitored, and the modifiable risk factors should be corrected (arterial hypertension, concomitant use of OAC and antiplatelet therapy) [4].

The aim of this study is to evaluate the risks and the benefits of oral anticoagulation treatment in non-valvular atrial fibrillation patients, using the $\mathrm{CHA}_{2} \mathrm{DS}_{2}$-VASc and HAS-BLED clinical scores, recommended by the latest guidelines.

\section{Material and method}

A retrospective observational study was conducted on 144 patients admitted to the $3^{\text {rd }}$ Medical Clinic of Tirgu Mures, between $1^{\text {st }}$ of July 2013 and 30 ${ }^{\text {th }}$ September 2013, with a 6 months follow-up.

Selection criteria included:

- Paroxysmal/persistent/permanent atrial fibrillation diagnosis present at discharge;

- Contact details in the patients chart, required for follow-up;

- Written consent from patients, for participating in the study.

Based on the data collected from the charts, the $\mathrm{CHA}_{2} \mathrm{DS}_{2}$-VASc and HAS-BLED scores were calculated for all patients.
At 6 months, the patients were contacted via telephone and questioned regarding the presence of any hospitalization in the last 6 months, the last INR values, the existence of thromboembolic or bleeding events.

Bleeding complications were divided as follows:

- major bleeding events: the need for hospitalization, decrease in the hemoglobin level with more than 2 $\mathrm{g} / \mathrm{l}$, or the need for blood transfusion. Intracranial hemorrhage was followed separately during this study.

- minor bleeding events: asymptomatic drop of hemoglobin level, that led to temporary cessation of OAC treatment (epistaxis, GI bleeds, hematuria)

Data was inserted in Microsoft Excel and Epi Info software was used for statistical calculus. Chi square and Fisher's exact test were used, with a confidence level of $95 \%$. A p value $<0.05$ was considered statistically significant.

\section{Results}

From the 144 patients included in the study, 70 (49\%) were female and $74(51 \%)$ were male. The mean age of all the patients was $70 \pm 11$ years (35-91 years) (Figure 1).

On admission, most of the patients presented with permanent AF (93 patients, representing 65\% from the total patients), followed by 28 patients with persistent $\mathrm{AF}$ (representing 19\%) and 23 patients with paroxysmal AF (representing 16\%).

The most frequent comorbidities present in the 144 patients were as follows: 116 patients presented with hypertension, 59 presented vascular diseases, 37 had diabetes mellitus associated, 13 presented a history of stroke and 11 presented a history of thromboembolism.

The $\mathrm{CHA}_{2} \mathrm{DS}_{2}$-VASc score was calculated for all the patients, and all 13 patients (11.7\%) presenting with a history of stroke were in the high risk class (Table III).

Arterial hypertension was the risk factor present in all patients who suffered a stroke, hypertension being statistically associated with the risk of stroke, $\mathrm{p}=0.0218$ and $\mathrm{OR}=$ 10.714 .

Heart failure and vascular disease were present in 10 out of the 13 stroke patients, most of them being male.

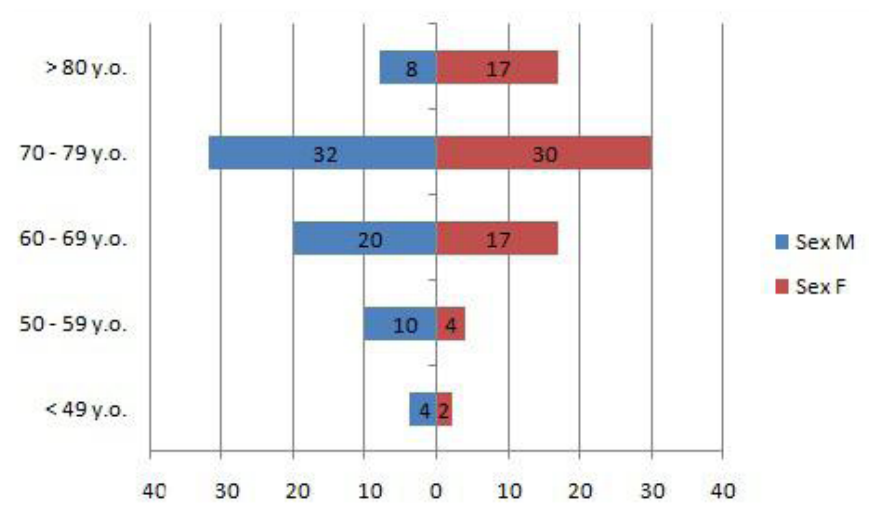

Fig. 1. Age and sex distribution of the studied group 
A statistically significant association was observed between vascular disease and stroke, $\mathrm{p}$ value $=0.0076, \mathrm{OR}=5.578$.

For every patient included in this study, the rate of bleeding complications was observed for a period of 6 months from admission. Each patient's HAS-BLED score was calculated, a score newly adopted by the guidelines, with the purpose to evaluate the hemorrhagic risk in patients on OAC treatment (Table IV).

Comparing the scores of the patients with and without a hemorrhagic event, the HAS-BLED score proved to be highly efficient in predicting a bleeding event in OAC patients, $\mathrm{p}=0.0003$.

From the total of 144 patients included in the study, $19(13.19 \%)$ presented bleeding complications during the 6 months of follow-up. Most of them presented with GI bleeding (10 patients representing 52.63\%), 7 patients presented macroscopic hematuria $(36.84 \%)$, complications that led to temporary cession of anticoagulant therapy (Figure 2).

The risk factors identified by us that could expose the anticoagulated patient to the possibility of bleeding event were: age over 65 years, history of bleeding $(\mathrm{p}=0.0001)$, history of stroke, anemia ( $\mathrm{p}=0.0451)$, female sex, uncontrolled hypertension, renal or liver insufficiency, labile INR values $(\mathrm{p}=0.0013)$, drugs and alcohol abuse.

From the total of 144 patients, in the 6 month period of follow-up, there were 5 deaths registered, representing $3.47 \%$. Death occurred mainly due to multiple comorbid states association. In one case, death occurred secondary to an ischemic stroke.

\section{Disscusions}

All patients who suffered a stroke were in the high class $\mathrm{CHA}_{2} \mathrm{DS}_{2}$-VASc score $\geq 2$ and they were not on OAC treatment at the time of the stroke, AF being diagnosed after the event. From the 13 stroke patients, 8 were male and 5 female, contrary to data from the literature. Many studies report a higher stroke rate in women, female sex being considered an independent risk factor [4,9-11]. One explanation of this founding could be the small number of patients studied by us.

In numerous cases, although there isn't a formal contraindication, the $\mathrm{OAC}$ treatment isn't initiated due to reduced compliance of the patient or due to the lack of treatment monitoring. According to other authors, only $15-44 \%$ of eligible patients benefit from OAC treatment [12].

Hypertension was the risk factor present in all stroke patients with a statistically significant association between the

Table III. Patients' distribution based on the $\mathrm{CHA}_{2} \mathrm{DS}_{2}$-VASc score

\begin{tabular}{lccc}
\hline CHA2DS2-VASc & & Patients, $n$ & Stroke \\
\hline Score $=0$ & Low risk & 1 & 0 \\
Score $=1$ & Moderate risk & 32 & 0 \\
Score $\geq 2$ & High risk & 111 & 13 \\
\hline
\end{tabular}

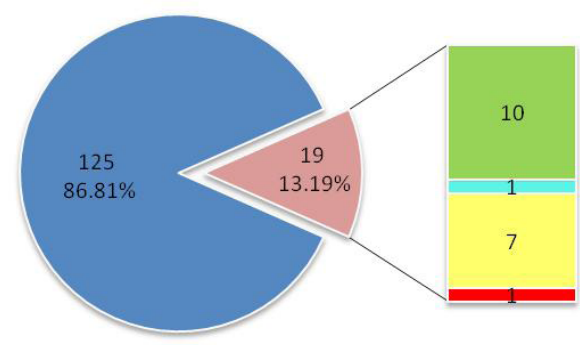

= No bleeding $\|$ GI bleeding $=$ Transfusion Hematuria $\|$ Hemorrhagic stroke

Fig. 2. Bleeding events rate in the studied group

two $(\mathrm{p}=0.0218$ and $\mathrm{OR}=10.714)$. Some authors consider that an adequate control of the blood pressure reduces the risk of stroke by $40 \%$, regardless of age [13].

Recurrence of thromboembolic events is an important factor to keep in mind, due to its increased morbidity and mortality in AF patients. Frequently, recurrences occur in the next days after a stroke, or in the next 30 days after a thromboembolic event. Some authors report a recurrence rate of $3.3 \%$ in the next 30 days from a stroke [14] and $25 \%$ in the next 5 years from the initial stroke [15].

Another important aspect that we followed during our study was the bleeding events from $\mathrm{OAC}$ treatment in $\mathrm{AF}$ patients. According to literature data, upper GI bleedings are the most frequent and the hospitalization rate is 11 fold higher due to them, than in the general population [16,17].

In the current study, most of the bleeding events occurred in the high risk patients with a HAS-BLED score $\geq$ 3; 16 of the 19 patients with bleeding events were in this category.

Labile INR values are associated with a higher risk of bleeding events. In our study 13 patients (68.42\%) presented with labile INR values from the total of 19 patients with bleeding complications. The statistical calculus revealed a p value of 0.0013 and an OR value of 5.356 (CI $95 \%=1.889-15.188)$, underlying an association between bleeding events and labile INR values. Many studies report a 2 fold increase of the bleeding risk in patients with INR values $\geq 3$ in comparison with patients whose INR values remain within the limits $[18,19]$. Another study reported a 2 fold increase of the risk of hemorrhage for every unit INR increases [20].

\section{Conclusion}

There are a series of common risk factors that contribute to thromboembolic events and bleeding complications in anticoagulated atrial fibrillation patients. Amongst them is

Table IV. Patients' distribution based on HAS-BLED score

\begin{tabular}{lcc}
\hline HAS-BLED score & On OAC & Bleeding events \\
\hline $0-1$ & 33 & 1 \\
2 & 47 & 2 \\
$\geq 3$ & 64 & 16 \\
Total & & 19 \\
\hline
\end{tabular}


uncontrolled hypertension, age higher than 65 years or a history of stroke.

The $\mathrm{CHA}_{2} \mathrm{DS}_{2}$-VASc clinical score is a useful tool in evaluating the risk of stroke in atrial fibrillation patients.

The HAS-BLED clinical score is useful in evaluating the patients before the initiation of antithrombotic therapy. Patients with a HAS-BLED score $\geq 3$ should be monitored cautiously, most of the patients in our study who suffered a bleeding complications being considered "high risk" patients.

\section{References}

1. ACC/AHA/ESC Guidelines for the Management of Patients With Atrial Fibrillation: Executive Summary A Report of the American College of Cardiology/American Heart Association Task Force on Practice Guidelines and the European Society of Cardiology Committee for Practice Guidelines and Policy Conferences (Committee to Develop Guidelines for the Management of Patients With Atrial Fibrillation). Circulation. 2001;104: 2118-2150.

2. Guidelines for the management of atrial fibrillation. European Heart Journal. 2010;31: 2369-2429.

3. Hart RG, Pearce LA, Aguilar MI. Meta-analysis: Antithrombotic therapy to prevent stroke in patients who have nonvalvular atrial fibrillation. Annals of Internal Medicine. 2007;146: 857-867.

4. Camm AJ, Lip GY, De Caterina R et al. Focused update of the ESC Guidelines for the management of atrial fibrillation: an update of the 2010 ESC Guidelines for the management of atrial fibrillation. European Heart Journal. 2012;33:2719-2747.

5. Pisters R, Lane DA, de Vos CB et al. A novel user-friendly score (HASBLED) to assess one-year risk of major bleeding in atrial fibrillation patients: The Euro Heart Survey. Chest. 2010;138(5):1093-1100.

6. Thrift AG, Donnan GA, Mc Neil JJ. Epidemiology of intracranial hemorrhage. Epidemiologic Reviews. 1995;17:361-381.

7. Kase CS. Intracerebral hemorrhage. Baillière's Clinical Neurology. 1995;4:247-278.

8. Shorr RL, Ray WA, Daugherty JR et al. Concurrent use of nonsteroidal anti-inflammatory drugs and oral anticoagulants places elderly persons at high risk of hemorrhagic peptic ulcer disease. Annals of Internal Medicine. 1993;153:1665-1670.

9. Eva Prescott, Rikke Sørensen. Female sex as a risk factor for stroke in atrial fibrillation. British Medical Journal. 2012;344:e3789.

10. Avgil TM, Jackevicius CA, Rahme E et al. Sex differences in stroke risk among older patients with recently diagnosed atrial fibrillation. The Journal of the American Medical Association. 2012;307:1952-1958.

11. Dagres N, Nieuwlaat R, Vardas PE et al. Gender-related differences in presentation, treatment and outcome of patients with atrial fibrillation in Europe: a report from the Euro Heart Survey on Atrial Fibrillation. Journal of the American College of Cardiology. 2007; 49:572-577.

12. Flaherty MLKB, Woo D, Kleindorfer D et al. The increasing incidence of anticoagulant-associated intracerebral hemorrhage. Neurology. 2007;68:116-121.

13. Geraint Fuller, Mark Manford. Neurology: An illustrated colour text, Churchill Livingstone Elsevier. Philadelphia. 2006;70-71.

14. Sacco RL, Foulkes MA, Mohr JP et al. Determinants of early recurrence of cerebral infarction: the Stroke Data Bank. Stroke. 1989;20:983-989.

15. Sacco RL, Shi T, Zamanillo MC et al. Predictors of mortality and recurrence after hospitalised cerebral infarction in an urban community: the Northern Manhattan Stroke Study. Neurology. 1994;44:626-634.

16. Hallas J, Dall M, Andersen BS et al. Use of single and combined antithrombotic therapy and risk of serious upper gastrointestinal bleeding: population based case-control study. British Medical Journal. 2006;333:726.

17. Sørensen R, Hansen ML, Abildstrom SZ et al. Risk of bleeding in patients with acute myocardial infarction treated with different combinations of aspirin, clopidogrel and vitamin K antagonists in Denmark: a retrospective analysis of nationwide registry data. The Lancet. 2009;374:1967-1974.

18. Saour JN, Sieck JO, Mamo LA et al. Trial of different intensities of anticoagulation in patients with prosthetic heart valves. New England Journal of Medicine. 1990;322:428-432.

19. Palareti G, Leali N, Coccheri S et al. Bleeding complications of oral anticoagulant treatment: an inception-cohort, prospective collaborative study (ISCOAT). Italian Study on Complications of Oral Anticoagulant Therapy. The Lancet. 1996;348:423-428.

20. Hylek EM, Singer DE. Risk factors for intracranial hemorrhage in outpatients taking warfarin. Annals of Internal Medicine. 1994;120:897902. 